\title{
Orientation variation of surface strain
}

\author{
R. Harder, ${ }^{1}$ M. A. Pfeifer, ${ }^{2}$ G. J. Williams, ${ }^{3}$ I. A. Vartaniants, ${ }^{4}$ and I. K. Robinson ${ }^{1}$ \\ ${ }^{1}$ London Center for Nanotechnology, Department of Physics and Astronomy, University College, London WC1E 6BT, United Kingdom \\ ${ }^{2}$ Department of Physics, La Trobe University, Victoria 3086, Australia \\ ${ }^{3}$ School of Physics, University of Melbourne, Victoria 3010, Australia \\ ${ }^{4}$ HASYLAB, DESY, Notkestrasse 85, D-22607 Hamburg, Germany
}

(Received 2 July 2007; published 20 September 2007)

\begin{abstract}
Expansion of the surface layers of a facetted hemispherical nanocrystal of $\mathrm{Pb}$ is reported at a temperature just below the melting point. Inversion of the coherent $\mathrm{x}$-ray diffraction pattern yields quantitative threedimensional maps of the deformation of the crystal from its equilibrium lattice spacing. Most of the surface of the crystal has a clear outward displacement, which decays exponentially into the bulk. This is suppressed on the (111) facet itself and is stronger on the spherical regions, suggesting that it arises from the orientational variation of the underlying surface stress.
\end{abstract}

DOI: 10.1103/PhysRevB.76.115425

PACS number(s): 61.46.Hk, 62.25.+g, 68.35.Gy, 81.10.Jt

Thermal expansion of metal surfaces at temperatures close to melting was investigated using ion scattering and x-ray diffraction methods in the 1980s (Refs. 1-5) and subsequently explained as due to enhanced anharmonicity. ${ }^{5-8}$ The interatomic potential experienced by an atom in a crystal surface, with no neighbors above it, is highly anharmonic. Because they have more degrees of freedom, surface phonons tend to be softer with typically lower frequency than the bulk. This results in greater vibration amplitudes at the surface, and hence, when coupled with the anharmonicity, leads to expanded interatomic spacings. Since this expansion can overcome the natural tendency of metal surfaces to contract in order to confine their valence electrons,,${ }^{9,10}$ it was termed "anomalous" when it was observed experimentally. ${ }^{3}$

According to the Lindemann model, ${ }^{11}$ a material melts when its vibration amplitudes become a significant fraction of the interatomic spacing, so the surface anharmonicity can cause surface melting, involving progressively greater number of layers as the melting point is approached. The phenomenon of surface melting has also been extensively studied and can be explained thermodynamically in terms of the surface energy. ${ }^{12}$ The crystal-orientation dependence of the surface energy explains why surface melting is minimized on the close-packed (111) facets and stronger in other directions. ${ }^{12}$ Surface melting leads to a "quasiliquid" layer, which retains much of the order of the underlying crystal lattice with additional thermally populated defects that can also contribute to the lattice expansion.

The Wulff construction, a Legendre transformation of the crystal-orientation dependence of the surface energy, explains how the equilibrium crystal shape (ECS) is spherical with facets. ${ }^{13,14}$ The facets are atomically flat, as seen in variable-temperature scanning tunneling microscopy experiments, ${ }^{15,17,19}$ while the spherical regions are rough in the sense that there are thermally excited kinks in the steps present in dynamic equilibrium. ${ }^{17}$ The ECS of $\mathrm{Pb}$ has been widely studied at temperatures close to melting ${ }^{13,15-18}$ and is known to contain only $\{111\}$ facets. ${ }^{13}$ The anisotropy of the surface free energy between the round regions with surface tension $\gamma_{0}$ and facets with $\gamma_{111}$ is $\gamma_{0} / \gamma_{111} \simeq 1.03$ (Refs. 13-16) at $T=573 \mathrm{~K}$, which gives rise to circular facets subtending an angle of $\tan ^{-1} \sqrt{2\left(\gamma_{0} / \gamma_{111}-1\right)}=14^{\circ}$. Since the sur- face stresses will, in general, be different between the flat and rough regions, there will be a pattern of strain expected inside the crystal, associated with the formation of an ECS. Continuum elasticity theory tells us that the resulting strain will take a form analogous to a Rayleigh wave that decays into the bulk over a distance comparable with the size of the facet. ${ }^{20}$ Recent works on the structure of surfaces containing step arrays ${ }^{21}$ and chemically induced variations of surface stress $^{22}$ have confirmed this strain distribution. From these considerations, we might expect to see an orientation dependence of the strain associated with the ECS. The observation of such a dependence is what we report here.

Coherent $\mathrm{x}$-ray diffraction (CXD) imaging is a powerful new method of three-dimensional (3D) lensless imaging of the interiors of small crystals. ${ }^{23}$ By exploiting the vastly improved coherence of undulator $\mathrm{x}$-ray radiation from third generation sources of synchrotron radiation, CXD methods rely on the mutual interference of waves scattered from all extremes of the crystal under investigation. The rich 3D diffraction pattern so produced can be sampled beyond its $\mathrm{Ny}-$ quist frequency, whereby it can be phased and, hence, inverted to a 3D image. Unless the diffraction pattern happens to be locally centrosymmetric, the resulting image is a $3 \mathrm{D}$ complex function. We recently demonstrated that, while the magnitude of this image represents the electron density, the phase of this complex function maps out the projection onto the momentum transfer vector $\mathbf{Q}$ of the deformations of the crystal from the underlying crystal lattice. ${ }^{24,25}$ The $3 \mathrm{D}$ images of a 750-nm-diameter $\mathrm{Pb}$ crystal were found to have a bump of negative phase protruding from the flat interface with the substrate, which was interpreted as due to the strain field arising from contact forces. ${ }^{24}$ Since the $\mathbf{Q}$ vector was pointing away from the interface, the negative sign of the phase arises from a compression of the crystal by the contact forces. Here, we report that the same nanocrystal also has a skin of positive phase on its outer surface, which we attribute to surface thermal expansion, and, furthermore, that the magnitude of the expansion has an interesting dependence on crystal orientation.

The CXD measurements are sensitive to strain because they were made with hard $\mathrm{x}$ rays at the large diffraction angle of the (111) Bragg peak. ${ }^{24}$ In the experimental result, the 
maximum observed phase shift was -1.4 rad relative to the bulk of the crystal located at a point near the center of the flat interface with the substrate. The rapid drop off of phase, moving away from the interface, was found to be consistent with the theory of continuum elasticity describing the spatial dependence of strain fields. ${ }^{20,25}$ The novelty of the information made available by the imaging technique is that the function describing the electron density of the interior of the solid crystal is allowed to be complex, rather than merely real. According to the Fourier theorem, the complex density manifests itself in noncentrosymmetry of the intensity distribution of the diffraction pattern. In the case of (111) Bragg diffraction from our $\mathrm{Pb}$ nanocrystal, this symmetry breaking is local to the mathematical Bragg point or center of the intensity distribution. The breaking of local symmetry can be explained as deviations from the ideal lattice underlying the crystal, most simply described as a field of displacements from the lattice. ${ }^{25}$

However, other contributions to the real-space phase must be considered as well. A recent study of a freeze-dried yeast cell also revealed a complex density function. ${ }^{26}$ In that case, the diffraction intensity data were asymmetric around the origin of reciprocal space, and so cannot be identified with strain. Indeed, a yeast cell contains no crystal lattice with respect to which strain could be defined. The authors of that study identified "non-Born approximation" scattering as the cause of broken symmetry. This is the same physical principle as optical refraction, which is usually ignored at x-ray wavelengths, but can be important for optically thick materials in certain wavelength ranges. ${ }^{27}$ In our original work, we also ignored the refraction effects in deference to the much more dramatic strain contribution, but here we correct for refraction in order to visualize further strain features in our original phase images.

Refraction causes the waves traveling inside the crystal to have a different wavelength from those traveling in the vacuum outside. For $8.9 \mathrm{keV} x$ rays used in our work, the real part of the refractive index $n$, being the ratio of these wavelengths, is less than unity by an amount $\delta=2.23$ $\times 10^{-5}$. The vacuum wavelength $\lambda=0.138 \mathrm{~nm}$ corresponds to a wave vector magnitude of $k=45.5 \mathrm{~nm}^{-1}$. The diameter of the $\mathrm{Pb}$ nanocrystal we studied was $d=750 \mathrm{~nm}$, over which distance the electromagnetic wave is retarded in phase, relative to a parallel one in vacuum, by $k d \delta=0.76 \mathrm{rad}$. This value, corresponding to a ray that traverses the thickest part of the crystal, is a significant fraction of the reported phase shift due to strain, $-1.4 \mathrm{rad}^{24}$ We note that the direct $\mathrm{x}$-ray absorption, $k d \beta$, is insignificant for a crystal of this size, being only $7 \%$, as given by the imaginary part of the refractive index, $\beta=2.19 \times 10^{-6}$ at $8.9 \mathrm{keV}$.

When Bragg diffraction takes place at some location inside the crystal, both the incoming $\mathbf{k}_{i}$ and outgoing $\mathbf{k}_{f}$ waves experience refraction. The (Bragg) angle between $\mathbf{k}_{i}$ and $\mathbf{k}_{f}$ was $28^{\circ}$ for the (111) reflection used here. The combined effect on our phase maps can be determined by considering the relative phase of the outgoing wave with respect to an identical copy of the crystal without refraction. A phase shift accumulates along both of these paths and so varies with position within the scattering volume. A spherical crystal of diameter $d=750 \mathrm{~nm}$ will have a phase shift of $\phi_{0}=k d \delta$
$=0.76 \mathrm{rad}$ for rays scattering from its exact center, the same as for the transmission case considered above. The phase shift is less for points above, and reaches a maximum value of $\phi_{0} / \cos 14^{\circ}=0.78 \mathrm{rad}$ at a point slightly below the center, where "up" and "down" are defined along or against the direction of the momentum transfer vector, $\mathbf{Q}=\mathbf{k}_{f}-\mathbf{k}_{i}$. We assume that the crystal used in our experiment was close to spherical in shape, and we ignore the cutoff on the bottom by the substrate plane because both $\mathbf{k}_{i}$ and $\mathbf{k}_{f}$ vectors necessarily lie above that plane.

We model the phase shift due to refraction using the distorted wave Born approximation. For scattering at a general point $\mathbf{r}$ inside the crystal, it is assumed that the refraction phase shift simply accumulates along the path of the incident wave vector $\mathbf{k}_{i}$. In a homogeneous medium, the incident beam is distorted by refraction but retains the structure of its wave front until it reaches point $\mathbf{r}$, whereupon it scatters into a spherical wave. The contributions from all points $\mathbf{r}$ within the crystal are detected along the exit wave direction $\mathbf{k}_{f}$. Each scattered wave experiences a phase shift due to refraction along its exit path. In the Born approximation, there is only one scattering event, so the path for a given $\mathbf{r}$ can be traced uniquely when $\mathbf{k}_{i}$ and $\mathbf{k}_{f}$ are known. In this way, we can assign a refraction phase shift to every point in the crystal. For a sphere, the resulting phase shift at a point $\rho$ in dimensionless units $\rho=\mathbf{r} / r_{0}$ as a fraction of the radius of the crystal, $r_{0}$, is given by

$$
\phi=\frac{\phi_{0}}{2} \sum_{j=i, f}\left(\hat{\mathbf{k}}_{\mathbf{j}} \cdot \rho+\sqrt{1+\left(\hat{\mathbf{k}}_{\mathbf{j}} \cdot \rho\right)^{2}-|\rho|^{2}}\right),
$$

where the $\hat{\mathbf{k}}$ 's are unit vectors pointing toward the center of the sphere in the directions of the incident and exit beams.

In our case, we know an exact $3 \mathrm{D}$ representation of the shape of the crystal from the previous analysis. ${ }^{24}$ Since the precise shape and complete information about the directions of $\mathbf{k}_{i}$ and $\mathbf{k}_{f}$ are known, the phase shift could be evaluated numerically without adjustable parameters. This function, which closely resembles the expression for a sphere given in Eq. (1) above, is plotted in the top panel of Fig. 1. There is no phase shift only for the points right on the (111) facet that lies in the direction of the $\mathbf{Q}$ vector. The phase shift (path length) mounts rapidly for points below this plane, reaching $50 \%$ of $\phi_{0}$ for depths below about $0.13 d$. The bulk of the crystal, away from this region, has a phase shift approaching the value $\phi_{0}$. Thus, most of the variation of phase due to refraction is located on the opposite side of the crystal from the previously studied phase bulge ${ }^{24}$ for which the interpretation is not much affected.

We note that the absolute phase of a scattering object does not usually need to be defined, because the diffraction amplitude, given by the magnitude of its Fourier transform, does not depend on the choice of origin. A logical choice would be to define the phase to be $\pi$ as this is the phase of the wave scattered by a point charge (electron) illuminated by a plane wave. ${ }^{27}$ In the absence of refraction, diffraction from all electrons on the crystal lattice of the sample would have the same $\pi$ phase shift relative to the incoming wave.

The phasing algorithms used to obtain the image in Ref. 

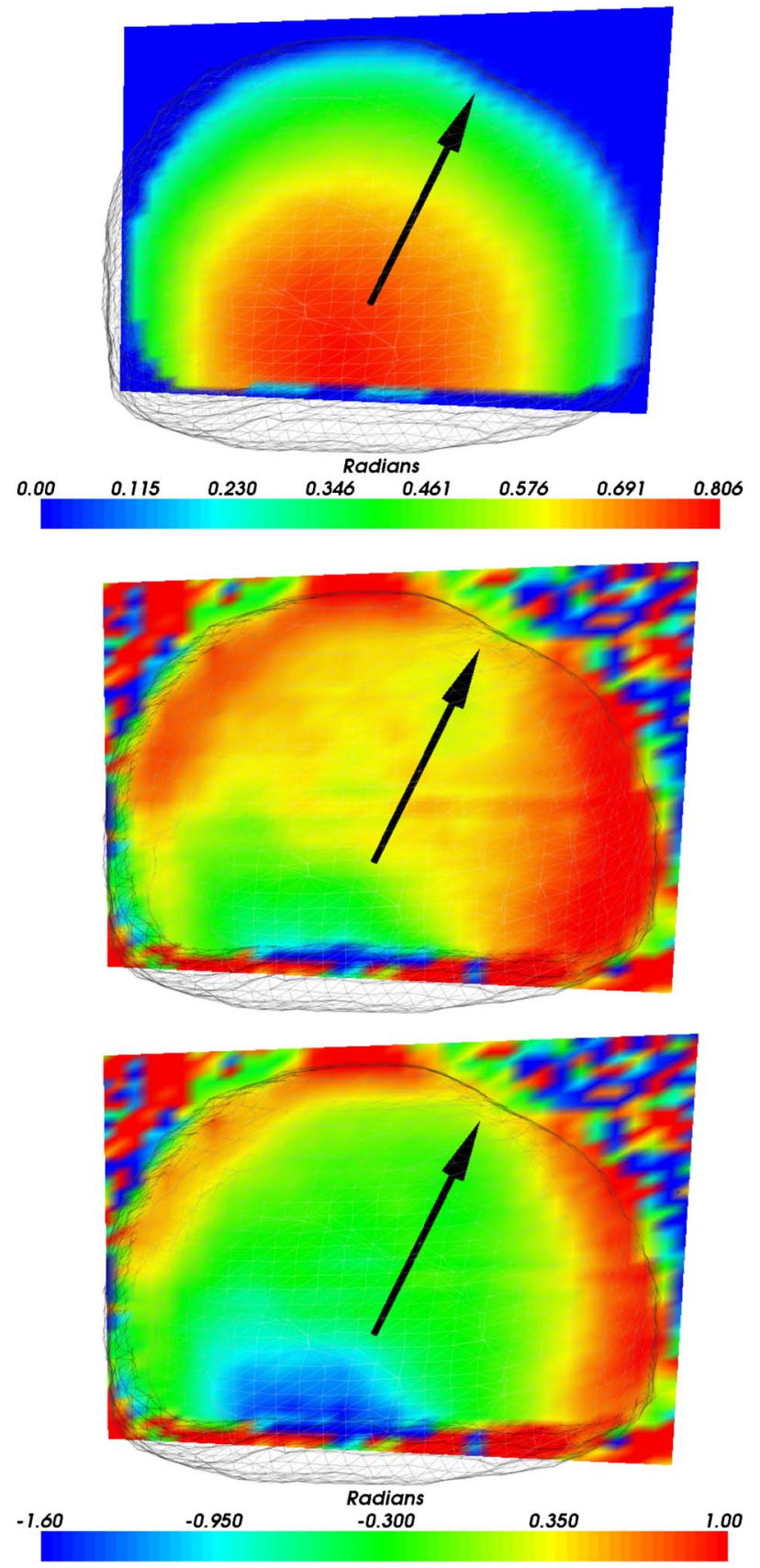

FIG. 1. (Color online) Sections through the center of the 3D complex density map passing through the (111) facet. The colors show the variation of the phase inside the nanocrystal, on the same scale, in the lower two panels. The arrow shows the direction of the (111) $\mathbf{Q}$ vector used for measuring the diffraction onto which the strain is projected. A 3D view of a single density contour $(50 \%$ $\max )$ is superimposed to show the shape of the crystal. Note that the phase becomes very noisy outside the crystal, where the amplitude of the complex density function vanishes. (Top) Refraction phase only; calculated from the shape of the crystal. (Middle) Phase map before correction for refraction. (Bottom) Phase map after correction for refraction.

24 employed cycles of Fienup's ${ }^{28}$ hybrid input output (HIO) method followed by support-constrained error reduction (ER). HIO utilizes a form of feedback based on previous iterates and the application of a support. To obtain complex images with a real-space phase function $\phi(\mathbf{r})$, we used a constraint of $-\pi / 2<\phi(\mathbf{r})<\pi / 2$ during the HIO cycles and no constraint on the phases during ER. This was considered to be a minimal constraint; with no constraints at all, the phases might tend to spin randomly, since the diffraction amplitude is independent of the absolute phase origin in real space. The chosen range had the consequence that the resulting image was centered with most of the phase values near $\phi(r)=0$.

In this paper, we examine the positive excursions of $\phi(r)$, which were localized at the "top" of the crystal, near where the $\mathbf{Q}$ vector emerges from the sphere. This is where most of the refraction effects are seen. The lower panels of Fig. 1 show the phase map before and after subtraction of the refraction phase. After correction, the positive phase structure becomes more pronounced and is clearly seen to follow the shape of the edge of the crystal, which is shown superimposed in Fig. 1 as a 50\% density isosurface. The (111) facet is clearly visible as a flat edge in this view. A least-squares fit to the shape of the crystal to a sphere and planes found six of the eight possible $\{111\}$ facets in addition to the flat interface that was noncrystallographic. ${ }^{24,29}$ The six $\{111\}$ facets subtended angles of $18.2 \pm 2.6^{\circ}$, roughly consistent with the $14^{\circ}$ determined previously for the ECS of $\mathrm{Pb}$ near this temperature. ${ }^{13}$ We focus our attention on the facet denoted (111) near the top of the crystal in the same direction as the

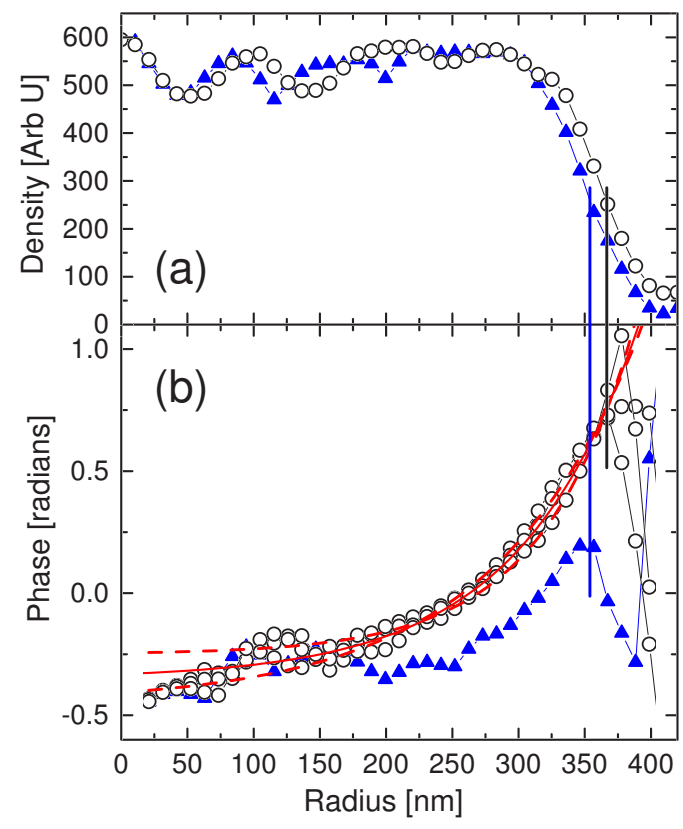

FIG. 2. (Color online) Line scans across the boundary of the crystal along the direction of the (111) facet (solid triangles) and across the spherical part of the surface in the vicinity of the facet, inclined at $20^{\circ}$ (black circles). Three different azimuths are shown in the lower panel. (a) Amplitude of the complex density function, representing the electron density, showing a resolution-limited stepfunction shape. The flat facet results in the density dropping at smaller radius (vertical lines). (b) Phase, proportional to the lattice displacement, showing a divergence. The fit curve (red solid line) is an exponential with decay length of $90 \mathrm{~nm}$. The extremes of the fitting range are denoted by red dashed curves with decay lengths of 70 and $110 \mathrm{~nm}$. 
measurement $\mathbf{Q}$ vector (arrow in Fig. 1). The phase structure is considerably weaker on this (111) facet than in the adjoining regions of the crystal.

The phase is the projection of the lattice displacement onto the $\mathbf{Q}$ vector, and so is sensitive to the normal component of strain here. The phase rises from the bulk of the crystal by $\Delta \phi=+1.15 \pm 0.1 \mathrm{rad}$ on the vicinal surface. This corresponds to a net displacement of the surface layers by $(\Delta \phi / 2 \pi) d_{111}=0.052 \mathrm{~nm}$ relative to the bulk. A positive phase implies an expansion of the crystal at its surface, which we attribute to the high-temperature anharmonicity of the lattice mentioned above. We believe the relative absence of strain on the facet itself $(+0.47 \mathrm{rad}$ or $0.02 \mathrm{~nm})$ is due to the compounding effect of stress relief within the ECS: if the facet underwent a lateral expansion and perpendicular contraction, the surrounding region would have a lateral contraction and perpendicular expansion. Superimposing the net expansion due to anharmonicity cancels the contraction on the facet and reinforces the expansion on the vicinal region, as found.

This pattern of alternating compression and expansion at a surface is found in the form of the Rayleigh wave solution of the continuum elasticity equations at a surface..$^{20}$ The distortions should die off exponentially inside the solid with a decay length of $(1 / 2 \pi) L$, where $L$ is the period of the wave along the surface. Here, we can identify $L \simeq 470 \mathrm{~nm}$, which is twice the diameter of the facet, so we would expect the strain to decay with a $1 / e$ depth of $75 \mathrm{~nm}$. Figure 2 shows the variation of the amplitude and phase inside the crystal along radial lines passing just outside the edge of the facet along three different azimuths inclined at $20^{\circ}$ to the facet normal. Outside the crystal where the electron density amplitude is small, the phase is not well defined. Inside, it follows an exponential curve with a fitted 1/e depth of $90 \pm 20 \mathrm{~nm}$, which is consistent with our expectation of $75 \mathrm{~nm}$.

In summary, we have applied a refraction correction to the 3D phase maps previously obtained by CXD for a 750-nm-diameter $\mathrm{Pb}$ nanocrystal. In addition to the negative phase bulge previously seen at the interface with the substrate, the corrected images have a skin of positive phase decorating the outer surface of the nanocrystal. The results are consistent with a general expansion of the surface, present in all directions except the (111) facet itself, which has a much reduced expansion. The depth of the strain field visualized is consistent with elastic relaxation of stresses arising from the formation of a facetted equilibrium crystal shape.

We thank V. Elser, M. Gillan, and S. Narasimhan for useful discussions. This research was supported by EPSRC Grant No. EP/D052939/1 and NSF DMR 0308660. The experimental work was carried out at the Advanced Photon Source (APS) beamline 34-ID-C, which was operated by the Materials Research Laboratory of the University of Illinois under DOE Contract No. DEFG02-91ER45439. APS is operated by DOE Contract No. W 31109 ENG 38.
${ }^{1}$ J. W. M. Frenken, F. Huussen, and J. F. van der Veen, Phys. Rev. Lett. 58, 401 (1987).

${ }^{2}$ G. Helgesen, D. Gibbs, A. P. Baddorf, D. M. Zehner, and S. G. J. Mochrie, Phys. Rev. B 48, 15320 (1993).

${ }^{3}$ P. Statiris, H. C. Lu, and T. Gustafsson, Phys. Rev. Lett. 72, 3574 (1994).

${ }^{4}$ K. Pohl, J.-H. Cho, K. Terakura, M. Scheffler, and E. W. Plummer, Phys. Rev. Lett. 80, 2853 (1998).

${ }^{5}$ A. Rühm, H. Reichert, W. Donner, H. Dosch, Ch. Grütter, and J. Bilgram, Phys. Rev. B 68, 224110 (2003).

${ }^{6}$ L. Yang and T. S. Rahman, Phys. Rev. Lett. 67, 2327 (1991).

${ }^{7}$ J. Xie, S. de Gironcoli, S. Baroni, and M. Scheffler, Phys. Rev. B 59, 970 (1999).

${ }^{8}$ S. Narasimhan, Surf. Sci. 417, L1166 (1998).

${ }^{9}$ R. Smoluchowski, Phys. Rev. 60, 661 (1941).

${ }^{10}$ M. W. Finnis and V. Heine, J. Phys. F: Met. Phys. 4, L37 (1974).

${ }^{11}$ F. A. Lindemann, Phys. Z. 11, 609 (1910).

${ }^{12}$ B. Pluis, A. W. Denier van der Gon, J. W. M. Frenken, and J. F. van der Veen, Phys. Rev. Lett. 59, 2678 (1987).

${ }^{13}$ J. C. Heyraud and J. J. Métois, Surf. Sci. 128, 334 (1982).

${ }^{14} \mathrm{M}$. Wortis, in Chemistry and Physics of Solid Surfaces, edited by R. Vanselow and R. Howe (Springer, Berlin, 1988), Vol. VII, p. 367.

${ }^{15}$ A. Emundts, M. Nowicki, and H. P. Bonzel, Surf. Sci. 496, L35 (2002).

${ }^{16}$ C. Bombis, A. Emundts, M. Nowicki, and H. P. Bonzel, Surf. Sci.
511, 83 (2002).

${ }^{17}$ K. Thürmer, J. E. Reutt-Robey, E. D. Williams, M. Uwaha, A. Emundts, and H. P. Bonzel, Phys. Rev. Lett. 87, 186102 (2001).

${ }^{18}$ M. Nowicki, A. Emundts, and H. P. Bonzel, Prog. Surf. Sci. 74, 123 (2003).

${ }^{19}$ K. Thurmer, E. Williams, and J. Reutt-Robey, Science 297, 2033 (2002).

${ }^{20}$ L. D. Landau and E. M. Lifshitz, Theory of Elasticity (Pergamon, Oxford, 1986).

${ }^{21}$ G. Prévot and B. Croset, Phys. Rev. B 74, 235410 (2006).

${ }^{22}$ B. Croset, Y. Girard, G. Prévot, M. Sotto, Y. Garreau, R. Pinchaux, and M. Sauvage-Simkin, Phys. Rev. Lett. 88, 056103 (2002).

${ }^{23}$ I. K. Robinson and J. Miao, MRS Bull. 29, 177 (2004).

${ }^{24}$ M. A. Pfeifer, G. J. Williams, I. A. Vartanyants, R. Harder, and I. K. Robinson, Nature (London) 442, 63 (2006).

${ }^{25}$ I. A. Vartanyants and I. K. Robinson, J. Phys.: Condens. Matter 13, 10593 (2001).

${ }^{26}$ D. Shapiro, P. Thibault, T. Beetz, V. Elser, M. Howells, C. Jacobsen, J. Kirz, E. Lima, H. Miao, A. M. Neiman, and D. Sayre, Proc. Natl. Acad. Sci. U.S.A. 102, 15343 (2005).

${ }^{27} \mathrm{~J}$. Als-Nielsen and D. McMorrow, Elements of Modern X-ray Physics (Wiley, New York, 2001).

${ }^{28}$ J. R. Fienup, J. Opt. Soc. Am. A 4, 118 (1987).

${ }^{29}$ M. A. Pfeifer, Ph.D. thesis, University of Illinois, 2005. 